\title{
Which Behaviors Do People Explain? A Basic Actor-Observer Asymmetry
}

\author{
Bertram F. Malle \\ University of Oregon
}

\author{
Joshua Knobe \\ Stanford University
}

\begin{abstract}
Attribution research has focused on how people explain behavior; the present paper examines which behaviors they explain. The authors introduce a framework that specifies when people wonder about and explain an event. Applied to behavior, the framework predicts which behavioral events (intentional vs. unintentional and observable vs. unobservable) actors and observers tend to explain: (a) Actors wonder more often about unintentional and unobservable behaviors, whereas observers wonder more often about intentional and observable behaviors. (b) In private explanations (directed to oneself), actors explain more unintentional and unobservable behaviors, whereas observers explain more intentional and observable behaviors. (c) In communicative explanations (directed to others), both actors and observers explain more intentional and observable behaviors. These hypotheses are supported in 5 studies using thought protocols, memory protocols, diaries, conversations, and novels.
\end{abstract}

The central question of classic attribution theory is how people explain social behavior (Heider, 1958; Jones \& Davis, 1965; Kelley, 1967). In this article, we focus on a different question: Which behaviors do people explain?

The classic approach of attribution research is best illustrated in one of its central findings: the actor-observer asymmetry (Jones \& Nisbett, 1972). The results of many studies have shown that actors and observers differ in how they explain a given behavior, with actors preferring situation causes and observers preferring person causes (see Watson, 1982). This comparison is based on the implicit assumption that actors and observers tend to explain the same behaviors to begin with. But do they? In this article we try to answer this question. We first introduce a general framework that specifies the psychological conditions that cause a person to explain why an event occurred. We then apply this framework to the domain of behavior explanations and predict specifically which behavioral events actors and observers will tend to explain.

\section{Theoretical Framework}

The framework consists of two elements: a distinction between two types of explanations and a theory of wonderings.

Bertram F. Malle, Department of Psychology, University of Oregon; Joshua Knobe, Department of Psychology, Stanford University.

This article is based partially on Bertram F. Malle's doctoral dissertation, which was completed at Stanford University. Portions of these results were presented at the American Psychological Society's meeting in June 1994 and in a dissertation award address at the meeting of the Society of Experimental Social Psychology in October 1995. We would like to thank Laura Carstensen, Herbert Clark, Leonard Horowitz, Lara London, Ronaldo Mendoza, and Rick Robins for helpful comments on drafts of this article

Correspondence concerning this article should be addressed to Bertram F. Malle, Department of Psychology, 1227, University of Oregon, Eugene, Oregon 97403. Electronic mail may be sent via the Internet to bfmalle@darkwing.uoregon.edu.
We first distinguish between the following types of explanations (cf. Ross \& Fletcher, 1985): People explain an event either (a) to themselves, because they wonder why it occurred ("private explanations") or (b) to another person, because they believe that this person is wondering why the event occurred ("communicative explanations").

Because both types of explanation are based on wonderings, an understanding of the psychological conditions that cause a person to explain an event requires an understanding of wonderings. We first present a theory of wonderings, briefly apply it to classic findings, and then derive our hypotheses for both private and communicative explanations of behavioral events.

\section{A Theory of Wondering Why}

We propose that at least three conditions must be satisfied for people to wonder why an event occurred: The person (a) is aware of the event, (b) is in a state of nonunderstanding, and (c) feels that the event's explanation is personally relevant.

Awareness. For people to wonder why an event occurred, they must first be aware of the event (i.e., notice, observe, or think about it). Lack of awareness can be due either to limited epistemic access (e.g., people rarely know the thoughts and feelings of others) or to limited attention (e.g., people rarely pay attention to their own gestures).

Nonunderstanding. Once people are aware of an event, they wonder why it occurred only if they think that they do not already have an explanation. They must be in a state of nonun derstanding (also referred to as a "knowledge gap"; Bromberger, 1965; Hilton, 1990; Hilton \& Slugoski, 1986). Note that the condition of nonunderstanding is a subjective one-for people to wonder why, they must believe they lack an explanation (even if in fact they do have one). Likewise, if people believe they have an explanation (even if in fact they do not), they will not wonder why.

Relevance. Once people are aware of an event and think they do not understand why it occurred, they may or may not care about their own lack of understanding. They will care about 
it, and be motivated to wonder why, if their state of nonunderstanding challenges a current or enduring goal (Jones \& Thibaut, 1958; Kruglanski, 1989), in particular the need for control and prediction (Heider, 1958; Miller \& Steinberg, 1975), for selfintegrity (Steele, 1988), or for conceptual coherence (Pepitone, 1968; Read \& Miller, 1994). A relevant challenge to one's understanding of an event can be pleasant, as in the state of curiosity (Berlyne, 1960); stressful, as in the state of dissonance (Festinger, 1957); or outright shattering, as in the case of trauma or illness (Janoff-Bulman, 1992; Taylor, 1983). In all of these cases, people who face challenges to their understanding are likely to wonder why the challenging event occurred. By implication, reaching an understanding of an event (i.e., finding an explanation) would, to some extent, reinstate control, predictability, self-integrity, and conceptual coherence.

These three conditions explain why negative events are so likely to elicit wonderings (Bohner, Bless, Schwarz, \& Strack, 1988; Wong \& Weiner, 1981): The condition of awareness is often satisfied because people quickly and strongly attend to negative events (Pratto \& John, 1991; Taylor, 1991); the condition of nonunderstanding is often satisfied because negative events contradict people's general expectation that good things will happen (Boucher \& Osgood, 1969; Taylor, 1991); and the condition of relevance is often satisfied because negative events typically challenge people's needs for control and prediction, self-integrity, or cognitive and affective balance (Taylor, 1991). Positive events, on the other hand, may be easily overlooked (violating awareness), may be readily understood with cultural and personal scripts (violating nonunderstanding), and seldom threaten people's needs for control or self-integrity (violating relevance).

The three conditions also explain why unexpected or surprising events are likely to elicit wonderings (Hastie, 1984; Pyszczynski \& Greenberg, 1981). The condition of awareness is often satisfied because inconsistent or surprising events are noticed and processed longer (e.g., Belmore, 1987; see Fiske \& Taylor, 1991 , for a review ); the condition of nonunderstanding is satisfied because surprising events by definition contradict a prior piece of knowledge; and the condition of relevance is often satisfied because surprising events may challenge one's current cognitive framework and make prediction or control more difficult. Expected events, on the other hand, are assimilated easily (violating awareness), can be readily understood with one's existing knowledge structures (violating nonunderstanding), and can confirm rather than threaten people's sense of control, prediction, and conceptual coherence (violating relevance).

This theoretical framework should apply to all types of events. In this article, we apply it specifically to behavioral events (doing, feeling, thinking, etc.) to answer our central question, namely, which behaviors actors and observers explain.

\section{Classification of Behavioral Events}

Which behavioral events can people choose from? Researchers have shown that verbs describing actions invite different explanations than verbs describing experiences ( $\mathrm{Au}, 1986$; Brown \& Fish, 1983; Fiedler \& Semin, 1988; Gilovich \& Regan, 1986; McArthur, 1972). Similarly, when observers switch their attention from a person's actions to his or her experiences, explanations change accordingly (Galper, 1976; Gould \& Sigall, 1977; Regan \& Totten, 1975; Wegner \& Finstuen, 1977). In analyzing which behaviors people explain, it therefore seems critical to differentiate between these two types of behavior. However, actions and experiences differ along two dimensions: intentionality and observability. Prototypical actions (e.g., "Ann invited Ben for lunch"') are both intentional and observable, whereas prototypical experiences (e.g., "Ben is excited") are both unintentional and unobservable. The importance of distinguishing between intentional and unintentional behavior has been emphasized by several attribution researchers (e.g., Buss, 1978; Heider, 1958; Jones \& Davis, 1965; Kruglanski, 1975; Locke \& Pennington, 1982; Malle \& Knobe, in press; White, 1991). The importance of distinguishing between observable and unobservable behaviors has been emphasized by research in personality judgments and self-perception (e.g., Funder, 1980; Funder \& Dobroth, 1987; John \& Robins, 1993; Kenrick \& Stringfield, 1980). We therefore examined the full $2 \times 2$ table of behavioral events, exploring jointly the dimensions of intentionality and observability (see Figure 1).

\section{Predicting Which Behaviors People Explain}

The strength of this two-dimensional classification becomes apparent when we apply the general theory of wondering to the specific case of actors and observers wondering about and explaining behavioral events. For these events, the general conditions of wondering (awareness, nonunderstanding, and relevance) yield separate predictions for the dimensions of intentionality and observability. We begin with the hypotheses about wonderings.

Hypothesis 1a. Actors tend to wonder more often about unintentional than intentional behaviors, whereas observers tend to wonder more about intentional than unintentional behaviors.

This hypothesis derives from the conditions of nonunderstanding and relevance. Actors usually think they know why they performed their intentional behaviors. Thus, they rarely are in a state of nonunderstanding with respect to their intentional behaviors, so they are unlikely to wonder about them. Observers, on the other hand, may find others' intentional behaviors more relevant than unintentional behaviors. For one, intentional behaviors are socially more consequential, being so tightly associated with issues of responsibility and blame (Shaver, 1985); moreover, they are highly diagnostic of a person's desires, beliefs, and skills (Malle \& Knobe, in press) as well as the per-

\section{Intentional Unintentional}

\begin{tabular}{|c|c|c|}
\cline { 2 - 3 } Observable & 1 & $\begin{array}{c}2 \\
\text { actions }\end{array}$ \\
Unobservable behaviors \\
\cline { 2 - 3 } & 3 & 4 \\
intentional thoughts & experiences \\
\hline
\end{tabular}

Figure 1. Classification of behavioral events. 
son's character (Jones \& Davis, 1965). Therefore, observers are more likely to wonder about intentional than unintentional behaviors.

Hypothesis $1 b$. Actors tend to wonder more often about unobservable than observable behaviors, whereas observers tend to wonder more about observable than unobservable behaviors.

This hypothesis derives from the awareness condition. Actors typically pay less attention to their own observable behaviors (e.g., facial expressions, gestures) than to their unobservable behaviors (e.g., sensations, thoughts, feelings). If actors do not pay attention to observable behaviors, they are unlikely to wonder about them. Observers, on the other hand, typically have less access to other people's unobservable behavior than to their observable behavior. If observers do not even have access to unobservable behaviors, they are unlikely to wonder about them.

The very same considerations allow us to derive parallel hypotheses about private explanations because these explanations are a direct result of one's own wonderings.

Hypothesis 2. When people explain a behavior because they themselves wonder why it occurred, actors tend to explain unintentional behaviors more often than intentional behaviors, whereas observers tend to explain intentional behaviors more often than unintentional behaviors (Hypothesis 2a). Furthermore, actors tend to explain unobservable behaviors more often than observable behaviors, whereas observers tend to explain observable behaviors more often than unobservable behaviors (Hypothesis 2b).

Finally, in communicative explanations, people explain an event because they think that another person (their communication partner) wonders why it occurred. Such communicative explanations range from answers to others' "why" questions (e.g., in interview situations) to spontaneously offered accounts (e.g., in conflict situations; cf. Scott \& Lyman, 1968). As observers, people will continue to explain intentional and observable events, such as when they are explaining a third party's behavior. Even as actors, they will explain those behaviors that their communication partner (qua observer) is or might be wondering about, which are intentional and observable events once more. In communicative explanations, therefore, both actors and observers explain more intentional and more observable events. This reasoning leads to Hypothesis 3 .

Hypothesis 3. When people explain a behavior because they believe that another person wonders why it occurred, they tend to explain, both as actors and observers, more intentional than unintentional behaviors and more observable than unobservable behaviors.

We tested these hypotheses in five studies by examining the distributions of actors' and observers' wonderings and explanations in thought protocols, memory protocols, diaries, natural conversations, and novels.

\section{Study 1}

In this study, we attempted to record naturally occurring wonderings to test Hypothesis 1. Because wonderings can spontaneously occur in one's mind at any time, we asked participants to keep thought protocols of their spontaneous wonderings for several days.

\section{Method}

Participants. Twenty-eight undergraduates in an introductory psychology class received course credit for keeping thought protocols of their wonderings.

Procedure. Participants were recruited through a sign-up sheet. A research assistant called the volunteers and explained the purpose of the study as follows:

We are interested in the circumstances under which people find themselves wondering about some behavior or experience. Wondering means, for example, when you try to understand why you are in a particular mood, or when you try to understand why your friend is angry at you. For our study, you just introspect a little during any normal day and take brief notes whenever you wonder about something. In the evening, you review your notes, select four events, and describe them in more detail in a questionnaire (two pages for each day).

Participants who agreed to participate were invited to a group instruction session a few days later. The instructions explained that wonderings are

mornents at which you notice a particular experience (e.g., bodily state, emotion, sensation) in yourself and wonder why you have that experience; moments at which you notice a particular behavior you showed and wonder why you showed that behavior; moments at which you notice somebody else having a particular experience (e.g., bodily state, emotion, sensation) and wonder why the person has that experience; moments at which you notice somebody else showing a particular behavior and wonder why the person shows that behavior

We want to know when these moments occur in your own mind and what kinds of things you wonder about (try to understand, try to explain). Among these things you wonder about, we are primarily interested in experiences or behaviors that concern people-yourself and others - rather than objective events. Questions like "Why can't we have world peace?" or "Why is it raining today?" may be interesting, but they are not the focus of our research. We are interested in how you come to understand yourself and other people and what kinds of things you try to understand about yourself and others.

Participants pursued their daily activities as usual but carried a small notebook in which they wrote a few key words about each wondering as it occurred. Later in the day (usually in the evening), they reviewed their notes, selected four events from that day, and completed a brief questionnaire in which they described these events in more detail. Each participant could participate for a total of 4 days, thus describing a maximum of 16 events.

Materials. Each participant received a $3 \times 5 \mathrm{in} .(7.6 \times 12.7 \mathrm{~cm})$ spiral notebook and a small pencil for taking notes on wonderings as they occurred. In the questionnaire that documented the daily wonderings in more detail, participants described (a) the circumstances (time, place, and events) under which a wondering occurred; (b) what they were wondering about; (c) whether they could answer their wondering question; and (d) if so, what the answer was.

Coding. The 28 participants described a total of 338 wonderings, which were coded for perspective (actor vs. observer), explanation type (private vs. communicative), and behavior types. The four behavior types resulted from crossing the dimensions of intentionality and observability ( see Figure 1) and were labeled as follows: Actions are observable and intentional (e.g., "Why did she call her mom right after getting back to school?"'). Mere behaviors are observable and unintentional (e.g., "Why is she blushing?"). Intentional thoughts are intentional 
Table 1

Wonderings About Behavioral Events in Study I (Thought Protocols)

\begin{tabular}{lccccccc}
\hline & \multicolumn{3}{c}{ Actor } & & \multicolumn{3}{c}{ Observer } \\
\cline { 2 - 3 } Behavior & Intentional & Unintentional & Total & & Intentional & Unintentional & Total \\
\hline Observable & 23 & 10 & 33 & 60 & 13 & 73 \\
Unobservable & 3 & 59 & 62 & 5 & 30 & 35 \\
Totals & 26 & 69 & & 65 & 43 & \\
\hline
\end{tabular}

and unobservable (e.g., "Why is he trying to think of another word?"). Experiences are unobservable and unintentional (e.g., "Why am I so tired?'). Of the 338 wonderings, 94 ( $28 \%$ ) had to be excluded because they did not provide a test of our hypotheses. Sixty-nine of these were not wonderings why - the focus of our study - but wonderings if (e.g., "I wondered if I would get my work done in time") or wonderings how (e.g., "I was wondering what it will be like to go home for summer vacation"). The remaining items were excluded because they contained passive voice, "we" (first-person plural) statements, or wonderings about nonbehavioral events. (In the Appendix, we discuss in detail all exclusion criteria.) An additional 36 wonderings referred to dispositions ("Why do I procrastinate so much?" or "Why do people put so much pressure on themselves?"); these were also excluded because our hypotheses apply to concrete behavioral events only. ${ }^{1}$ Finally, five events could not be classified uniquely as one of the four behavior types; in four of the five cases, an observer wondered about an observable event whose intentionality was left ambiguous (e.g., "I was in a store and I was wondering why the clerk wasn't approaching me to offer me assistance"). After these exclusions, 203 codable events remained: 95 from the actor perspective, 108 from the observer perspective.

Reliability was determined by selecting wonderings from one fourth of the participants ( 7 participants and 78 events) and having two coders code the events for codability, perspective, and behavior type. After attending a training session based on a written coding instruction (see the Appendix ), the coders worked separately on the seven questionnaires. Their initial agreement on codability was $92 \%$. Among the events they both found codable, their initial agreement on perspective was $100 \%$, and on behavior type it was $74 \%$ (agreement on observability alone was $89 \%$, and on intentionality alone it was $87 \%$ ). These 50 cases on which coders agreed perfectly served as model cases for the remaining events, which were coded only by one coder.

\section{Results and Discussion}

Table 1 shows the distribution of 203 wonderings across the four behavior types for actors and observers. Both parts of Hypothesis 1 were confirmed: (a) Actors wondered more often about unintentional (73\%) than intentional (27\%) behaviors, whereas observers wondered more often about intentional (60\%) than unintentional ( $40 \%$ ) behaviors. In a three-way loglinear $(N=203)$ analysis, the corresponding interaction between perspective and intentionality was significant, partial $\chi^{2}(1)=4.2, p<.05$. (b) Moreover, actors wondered more often about unobservable $(65 \%)$ than observable $(35 \%)$ behaviors, whereas observers wondered more often about observable (68\%) than unobservable (32\%) behaviors. The corresponding interaction between perspective and observability was significant as well, partial $\chi^{2}(1)=4.0, p<.05$. In addition, $85 \%$ of all wonderings referred to actions (Behavior Type 1 ) or experiences
(Behavior Type 4), resulting in a two-way interaction between intentionality and observability, partial $\chi^{2}(1)=94.8, p<.001$.

We also analyzed the data using participants as the unit of analysis. This analysis determined how many participants would conform to the specified hypotheses. To that end, the target hypotheses were reformulated as follows: In wonderings from the actor perspective, a given participant should wonder more often about unintentional than intentional events (actor-unintentionality hypothesis ) and more often about unobservable than observable events (actor-unobservability hypothesis). In wonderings from the observer perspective, a given participant should wonder more often about intentional than unintentional events (observer-intentionality hypothesis) and more often about observable than unobservable events (observer-observability hypothesis ).

Each participant's set of wonderings was arranged into two $(2 \times 2)$ behavior-type tables, one for the actor perspective and one for the observer perspective. Then each table was classified as either conforming to the aforementioned hypotheses, contradicting them, or showing a "tie."

For the actor perspective, 22 participants provided usable data. Among them, 15 conformed to the actor-unintentionality hypothesis (binomial test, $p<.02$ ), with 6 contradicting and 1 tie. Moreover, 13 conformed to the actor-unobservability hypothesis $(p<.07)$, with 7 contradicting and 2 ties. For the observer perspective, 25 participants provided usable data. Among them, 15 conformed to the observer-intentionality hypothesis $(p<.02)$, with 6 contradicting and 4 ties. Moreover, 19 conformed to the observer-observability hypothesis $(p<$ .001 ), with 4 contradicting and 2 ties.

The results of this study support Hypothesis 1: Actors wondered more often about unintentional and unobservable behaviors, whereas observers wondered more often about intentional and observable behaviors. We tested Hypotheses 2 and 3 in Study 2.

\section{Study 2}

In this study we tested Hypotheses 2 and 3 (regarding private and communicative explanations) by examining memory proto-

\footnotetext{
${ }^{1}$ Dispositions would be difficult to code because they have ambiguous observability and ambiguous intentionality. Considered as internal mechanisms, they are unobservable and usually unintentional (e.g., procrastination), but when considered as (recurring) behavioral instantiations ( see Buss \& Craik, 1983), they can be observable and sometimes intentional (e.g., "Yesterday I procrastinated because I preferred to see a movie"). Explanations of dispositions, albeit rare, should be examined in more detail in future research.
} 
cols of behavior explanations. Participants described a recent instance in which they explained their own behavior (actor perspective) and another instance in which they explained someone else's behavior (observer perspective). These descriptions then were coded for explanation type (private vs. communicative) and behavior type. The protocols were collected using two similar forms, one for each of two samples. Any differences are noted.

\section{Method}

Participants. The participants in Sample A were 119 undergraduates in an introductory psychology class who received class credit for participating in the study. Nine participants did not complete the questionnaire or misunderstood the instructions, leaving a sample of 110 .

The participants in Sample B were 173 undergraduates in an introductory psychology class, who received course credit for participating in the study. Twenty-three participants did not complete the questionnaire or misunderstood the instructions, leaving a sample of 150 .

Procedure. Form A of the memory protocols was administered as part of a larger survey during a mass testing session. The participants were tested in groups of 5-20. The experimenter handed each student a booklet with all instructions and measures: As each student finished the booklet, the experimenter thanked and debriefed that student. Form B was administered as part of a larger survey in a take-home mass testing. The participants picked up a survey in class, filled it out at home, and returned it within 3 days.

Materials. The surveys contained detailed instructions for participants to report about events that they had tried to explain from each of the two perspectives (actor and observer). The instructions, which were identical for the two forms except for slight differences in wording, read as follows:

1. Please remember the last time you tried to explain somebody else's behavior or experience - for example, why the person acted a certain way, why the person felt a certain way, why something happened to them.

2. Please remember the last time you tried to explain your own behavior or experience-for example, why you acted a certain way, why you felt a certain way, why something happened to you.

All participants received both perspective instructions, and the order was counterbalanced across participants. Similarly, the order of the objects of explanation ("behavior or experience") and the order of the first two examples ("acted a certain way" and "felt a certain way") were counterbalanced across participants.

After each perspective instruction, participants answered three questions, each of which was followed by two empty lines:

(1) Please describe the circumstances in which you provided the explanation (e.g., to yourself in "silent talk" or to another person during a conversation).

(2) Please specify what you were trying to explain.

(3) Please write down (verbatim, if possible) the explanation you gave.

Coding of responses. The 110 participants of Sample A generated 212 behavior descriptions overall. The 150 participants of Sample B generated 285 behavior descriptions overall. The descriptions from each form were typed up and assembled in two small coding booklets. The following are two examples of behaviors that people explained (with their explanations in parentheses): "Why my friend left without telling. (Maybe he had an emergency or needed to get gas.)" "Why I became upset and frustrated with my boyfriend. (I felt challenged by a question.)"

Two coders coded the material in the booklets on the following variables ( see the Appendix); codability (whether the response was a behavior explanation or not), perspective (whether an event was explained from the actor perspective or from the observer perspective), explanation type (private or communicative), and behavior type (intentionality and observability).

After attending a training session based on the written coding instruction ( see the Appendix), the coders separately worked through the two booklets. Their initial agreement on codability was $92 \%$ for Sample A and $96 \%$ for Sample B. Among the events they both found codable, initial agreement (for each sample, respectively) was as follows: for perspective, $98 \%$ and $98 \%$; for explanation type, $90 \%$ and $93 \%$; and for behavior type, $81 \%$ and $84 \%$. The agreement on behavior type was greater for observability alone ( $94 \%$ and $96 \%$ ) than for intentionality alone ( $85 \%$ and $88 \%$ ). After discussing all cases in which they had disagreed, the coders' final agreement increased for codability (98\% and $98 \%$ ), perspective ( $100 \%$ and $100 \%)$, explanation type ( $96 \%$ and $98 \%$ ), and behavior type ( $94 \%$ and $93 \%$ ). The explanations on which they disagreed even after discussion were excluded from further analysis.

Of the resulting 199 explanations in Sample A, the coders agreed to exclude $28(14 \%)$, leaving 171 explanations for analysis. About half were excluded because their type (private vs. communicative) could not be determined; the remaining exclusions were "we" statements or phrased in the passive voice ( see the Appendix). Of the 285 explanations in Sample B, the coders agreed to exclude 80 (28\%), leaving 205 for analysis. Forty-one explanations had to be excluded because the participants did not identify the explanation type (private or communicative) in which the explanation occurred; 20 explanations did not explain why a behavioral event occurred but described that or how it occurred; the remaining ones did not refer to human behaviors, were "we" statements, and so on (see the Appendix). In initial analyses, Samples A and $B$ produced identical results and were therefore pooled, making a total sample of 376 explained behaviors.

\section{Results and Discussion}

The distribution of the 376 behaviors is displayed in Table 2. (All analyses were performed using events as the unit of analysis.) To test the two target hypotheses, we performed two three-way log-linear analyses (one for each explanation type), with perspective, observability, and intentionality as the three variables.

When giving private explanations, actors explained more unintentional $(73 \%)$ than intentional $(27 \%)$ behaviors, whereas observers explained more intentional $(67 \%)$ than unintentional (33\%) behaviors, supporting Hypothesis $2 \mathrm{a}$. The corresponding interaction between intentionality and perspective was significant, partial $\chi^{2}(1)=10.0, p<.002$. Moreover, actors explained somewhat more unobservable (54\%) than observable $(46 \%)$ behaviors, whereas observers explained more observable $(81 \%)$ than unobservable (19\%) behaviors, supporting Hypothesis $2 \mathrm{~b}$. The corresponding interaction between perspective and observability was significant, partial $\chi^{2}(1)=5.0, p<.03$.

When giving communicative explanations, actors' and observers' patterns of explanations were similar. Both explained somewhat more intentional $(55 \%)$ than unintentional $(45 \%)$ behaviors (although this trend was not significant), and both explained more observable $(69 \%)$ than unobservable $(31 \%)$ behaviors, partial $\chi^{2}(1)=29.9, p<.001$. However, this preference for observable behaviors was greater for observers $(82 \%)$ 
Table 2

Private and Communicative Explanations of Behavioral Events in Study 2 (Memory Protocols)

\begin{tabular}{|c|c|c|c|c|c|c|}
\hline \multirow[b]{2}{*}{ Behavior } & \multicolumn{3}{|c|}{ Actor } & \multicolumn{3}{|c|}{ Observer } \\
\hline & Intentional & Unintentional & Total & Intentional & Unintentional & Total \\
\hline \multicolumn{7}{|c|}{ Private explanations } \\
\hline Observable & 21 & 21 & 42 & 56 & 14 & 70 \\
\hline Unobservable & 4 & 45 & 49 & 2 & 15 & 17 \\
\hline Totals & 25 & 66 & & 58 & 29 & \\
\hline \multicolumn{7}{|c|}{ Communicative explanations } \\
\hline Observable & 48 & 13 & 61 & 51 & 25 & 76 \\
\hline Unobservable & 8 & 36 & 44 & 1 & 16 & 17 \\
\hline Totals & 56 & 49 & & 52 & 41 & \\
\hline
\end{tabular}

than for actors $(58 \%)$, producing a significant interaction between perspective and observability, partial $\chi^{2}(1)=16.8, p<$ .001 . This difference in observable behaviors might have occurred because, even in communicative contexts, actors are not aware of all their observable behaviors (but they are aware of their unobservable behaviors). They may try hard to cater to the observer's needs, but they do so within epistemic constraints. Moreover, in intimate conversations (which many of the reported ones were), partners may explain more of their own experiences than in nonintimate conversations. (This issue is developed further in the General Discussion section.)

Across perspective and explanation type, participants explained mainly actions (observable and intentional) and experiences (unobservable and unintentional); these two behavior types alone made up more than three fourths of all remembered explanations. (The corresponding two-way interaction between observability and intentionality was significant in both explanation types, $p \mathrm{~s}<.001$.)

The results of Study 2 generally support the two target hypotheses on people's private and communicative explanations. However, the data deviate slightly from the predictions, thus demanding further tests. The following two studies provided such tests, using more direct and naturalistic methods to examine people's explanations. Instead of coding the two types of explanation post hoc, we collected private and communicative explanations in separate contexts. In Study 3 we examined personal diaries to test actor-observer differences in private explanations (Hypothesis 2). In Study 4 we examined actual conversations to test actor-observer differences in communicative explanations (Hypothesis 3 ).

\section{Study 3}

Personal diaries, which document the vicissitudes of everyday experience, are likely to contain private explanations (i.e., explanations answering one's own wonderings). In this study, we collected seven diaries, selected passages that contained explanations of behavioral events, and coded them for perspective and behavior type to test Hypothesis 2 .

\section{Method}

Participants and procedure. The participants were 7 undergraduates from introductory psychology classes. Together, they provided more than 600 pages of personal diaries in exchange for course credit. Participants were recruited through an advertisement posted on the class bulletin board, inviting them to make their diaries available for study. Participants were assured of their complete anonymity (no demographic information was collected) and of the confidentiality of the diaries' contents. A research assistant collected each diary, copied its contents, returned it to the participant, and debriefed that participant. Two coders then selected wonderings and explanations from the copied diaries and coded them according to the following procedure.

Coding. From more than 600 pages of diaries, 322 passages were selected that contained linguistic markers of explanations (e.g., because, 'cos, in order to, due to, so that) or wonderings (e.g., why). The selected passages were printed in a booklet and given to two independent coders. The passages were coded for three variables: codability, perspective, and behavior type. The coders' initial agreement on codability was $82 \%$. Among the events they both found codable, their agreement on perspective was $100 \%$, their agreement on behavior type was $87 \%$ (for observability alone it was $95 \%$, and for intentionality alone it was $92 \%$ ). After discussing their disagreements, the coders agreed that 213 passages were codable ( 6 remaining disagreements were ruled uncodable). A considerable number of passages $(n=98)$ had to be excluded because many natural uses of "because," "cos," and so on do not express behavior explanations (of the 91 excluded passages, 24 were claim backings, 16 were nonbehavioral explanations, 14 were "we" statements, and the rest were dispositions, quoted explanations, normative statements, hypotheticals, or abilities; see the Appendix ). Likewise, many natural uses of "why" do not express behavior wonderings (of the 13 excluded passages, 6 were normative, 4 were statements of the form "That's why ...." and 3 concerned nonbehavioral events). In addition, five explanations occurred in a rough draft of a letter, thus being communicative by nature. They were excluded because such a small number did not allow comparisons between private and communicative explanations. The final 213 passages contained 172 explanations and 41 wonderings.

Wonderings and private explanations should produce the same pattern of behaviors (i.e., Hypotheses $I$ and 2 are equivalent). Indeed, an initial log-linear analysis on passage type (explanation vs. wondering), perspective, observability, and intentionality showed that no relevant effect involving passage type was significant. To increase power, we collapsed the data over the two types. 


\section{Results and Discussion}

Table 3 shows the distribution of 213 behaviors separately for actors and observers. The results clearly support Hypothesis 2a: Actors explained more unintentional $(75 \%)$ than intentional $(25 \%)$ behaviors, whereas observers explained more intentional (62\%) than unintentional ( $38 \%$ ) behaviors. In a three-way loglinear analysis $(N=213)$ the corresponding interaction between perspective and intentionality was significant, partial $\chi^{2}(1)=$ $5.0, p<.05$. Similarly, the results support Hypothesis $2 \mathrm{~b}$ : Actors explained more unobservable ( $78 \%$ ) than observable $(22 \%)$ behaviors, whereas observers explained more observable (59\%) than unobservable $(41 \%)$ behaviors. The corresponding interaction between perspective and observability was significant, partial $\chi^{2}(1)=5.4, p<.05$.

The results of Study 3 thus support Hypothesis 2 regarding actors' and observers' different behaviors they select in private explanations. The results of Study 3 replicate those of Study 2, but they differed in one respect. When diary writers took the observer perspective, they explained a greater number of experiences $(27 \%)$ than observers did in Study $2(17 \%)$. This difference probably stemmed from the general experiential focus of writing in a diary. Consistent with this assumption, actors, too, explained more experiences in the diaries $(67 \%)$ than in the memory protocols of Study $2(51 \%)$. Despite this minor difference, the data of Study 3 clearly replicate those of Study 2, and together the two studies provide good support for Hypothesis 2 . We next tested Hypothesis 3, which concerns actors' and observers' communicative explanations.

\section{Study 4}

In conversations people do not merely deliver messages to each other; they try to understand their partner's utterances and clarify their own. This coordinated and shared activity of understanding and clarifying has been called grounding (e.g., Clark \& Brennan, 1991). Grounding means that, at every turn, speaker and addressee try to reach the mutual belief that the speaker's meaning was understood by the addressee (Clark \& Schaefer, 1989).

Grounding occurs on many levels, from the phonetic to the lexical and from words to intentions. To communicate successfully, both partners need to know what the other is up to. They therefore ground the meaning of their words as well as the meaning of their behavior.

In a communicative context, actors often ground the meaning of their own behavior by offering explanations of those behavioral events that their conversation partner (as observer) is most interested in, namely, intentional and observable behaviors. For the purpose of grounding, actors thus deviate from their usual pattern of explanations, as predicted by Hypothesis 3. In Study 4 we tested this hypothesis by selecting communicative explanations from transcribed conversations and coding them for perspective and behavior type.

\section{Method}

Materials. Explanations were selected from Book 1 of the LondonLund corpus, a linguistic corpus collected by the Survey of English Usage, University College London (Svartvik, 1990; Svartvik \& Quirk, 1980). The transcribed conversations were intended to represent spontaneous conversation among educated speakers. Most of the speakers are British and college educated. The recordings were made without the prior knowledge of the main participants, but consent to use the transcripts for research purposes was acquired afterward. Our explanations were selected from Book 1 of the corpus, which contains 14 conversations (about 119,000 words), recorded between 1964 and 1976, among male and female students, staff, and faculty (aged 21-60 years).

Selection and coding. The transcript of Book 1 was available as a computer file, allowing the systematic selection of potential explanations using linguistic markers. The markers used (and the number of selected text passages that contained them) were as follows: because (226), 'cos (82), since (4), out of (3), due to (1), (in order) to (22), so that (4). For purposes of comparison, we also selected potential wonderings using the marker "why" (56).

The initial file of selections contained 398 passages that were printed as a booklet and handed to two coders. To increase coding reliability, a sufficient text section surrounding each selection was provided, ranging from 10 to 100 words. To remove ambiguity, coders could also access the complete text file of Book 1.

Coders followed the usual instructions (see the Appendix) and coded each selection for codability, perspective, and behavior type. Initial agreement on the codability of passages was $91 \%$. Among the passages both found codable, agreement on perspective was $100 \%$, and agreement on specific behavior type was $91 \%$ (on observability alone it was $94 \%$, and on intentionality alone it was $97 \%$ ). The coders discussed all passages that had produced disagreement and sometimes converged on a code. If discussion did not lead to convergence, the passage was ruled uncodable. The coders had to exclude several passages because they did not express behavior explanations or behavior wonderings ( see the Appendix). Of the 56 "why" passages, 25 were uncodable because they did not refer to behavioral events; 7 because they were normative, passive, or dispositions; and 8 because they used "why" not to wonder but to describe a consequence ("That's why 1 didn't join you"). Of the 342 "because," "“cos," and so on passages, the uncodable ones included 102 claim backings ("It was odd because . . ."); 51 ambiguous or nonbehavioral events ("The concert couldn't start because ..."); 18 hypotheticals ("I wouldn't do that because . . ."); 13 ambiguous agents ("we" or generalized "you"); 11 quoted explanations

Table 3

Private Explanations of Behavioral Events in Study 3 (Diaries)

\begin{tabular}{lccccccc}
\hline & \multicolumn{3}{c}{ Actor } & & \multicolumn{3}{c}{ Observer } \\
\cline { 2 - 3 } \multicolumn{1}{c}{ Behavior } & Intentional & Unintentional & Total & Intentional & Unintentional & Total \\
\hline Observable & 26 & 14 & 40 & 16 & 4 & 20 \\
Unobservable & 19 & 120 & 139 & 5 & 9 & 14 \\
Totals & 45 & 134 & & 21 & 13 & \\
\hline
\end{tabular}


Table 4

Communicative Explanations of Behavioral Events in Study 4 (Natural Conversations)

\begin{tabular}{lccccccc}
\hline & \multicolumn{3}{c}{ Actor } & & & \multicolumn{3}{c}{ Observer } \\
\cline { 2 - 3 } Behavior & Intentional & Unintentional & Total & & Intentional & Unintentional & Total \\
\hline Observable & 38 & 4 & 42 & 37 & 3 & 40 \\
Unobservable & 12 & 14 & 26 & 3 & 13 & 16 \\
Totals & 50 & 18 & & & 40 & 16 & \\
\hline
\end{tabular}

("And I said, "because I would like to go with you' . .."); 18 normative, passive, process, or ability statements (see the Appendix); and 5 on which coders disagreed. The resulting codable set contained 124 behavior explanations and 16 wonderings.

\section{Results and Discussion}

We first inspected the small number of wonderings in these conversations. As expected, actors expressed wonderings only about unintentional behaviors ( 5 of $5 ; 3$ of these were unobservable). By contrast, observers wondered mainly about intentional behaviors ( 9 of $11 ; 7$ of these were observable). Consistent with our hypotheses, wonderings in a conversation show the same basic actor-observer asymmetry as wonderings in general.

By contrast, Table 4 shows that this asymmetry disappeared for the 124 communicative explanations: To their conversation partner, both actors and observers explained more intentional $(73 \%)$ than unintentional ( $27 \%$ ) behaviors and more observable $(66 \%)$ than unobservable ( $34 \%$ ) behaviors, thus supporting Hypothesis 3. As in the previous studies, actions (Behavior Type 1) and experiences (Behavior Type 4) accounted for $82 \%$ of all explained events, producing an interaction between observability and intentionality, partial $\chi^{2}(1)=49.7, p<.001$. Most importantly, however, in a three-way (Perspective $\times$ Intentionality $\times$ Observability) analysis, no effects involving perspective were significant $(p>.10)$, whereas the main effects, across perspective, for observability, $\chi^{2}(1)=13.1$, and intentionality, $\chi^{2}(1)=26.2$, were highly significant $(p<.001)$.

Consequently, a log-linear model consisting only of the Intentionality $\times$ Observability interaction and the two main effects (for observability and intentionality) already fit the data well, deviation $\chi^{2}(4)=5.97, p>.20$. The addition of actor-observer asymmetries (Perspective $x$ Intentionality and Perspective $x$ Observability interactions ) did not significantly improve the fit, $\chi_{\text {change }}^{2}(3)=3.83, p>.30$. Although the questionnaire-assessed explanations of Study 2 showed slight deviations from the prediction of Hypothesis 3, the naturalistic data of this study fully confirmed it.

To summarize, in conversations actors reversed their pattern of explanations from the usual dominance of unintentional and unobservable behaviors to a dominance of intentional and observable behaviors. The likely reason for this reversal is that actors' communicative explanations have a much different function than private explanations. In communication, actors explain behavior, not to answer their own wonderings but to respond to their partners' wonderings, thus assisting in the grounding of interpersonal behavior.
So far, we have examined natural and experimental data on people's folk explanations of behavior and identified several principles that govern which behaviors people explain. If these principles have generality, we ought to find evidence for them in works of literature as well. Great writers, just like ordinary people, must adhere to the principles of folk explanation. We therefore selected three novels from 20th-century English literature to examine which behaviors fictional characters try to explain. Because Studies 3 and 4 replicated the patterns of actual explanations first demonstrated in Study 2 , we designed Study 5 to replicate the pattern of wonderings demonstrated in Study 1.

\section{Study 5}

We selected novels by Virginia Woolf and James Joyce because they are known for their psychological descriptions of behavior and experience (Kiely, 1980), thus likely yielding a considerable number of behavior wonderings. All three novels were available as computer text files in a Stanford University database that offers numerous classic works of English literature on CD-ROM. ${ }^{2}$

\section{Method}

Materials. The novels selected were Virginia Woolf's (1927) To the Lighthouse (TL), Virginia Woolf's (1925) Mrs. Dalloway (MD), and James Joyce's (1916/1993) Portrait of the Artist as a Young Man (PA).

Procedure. To find passages containing wonderings, we searched the text body of each novel (using tailored software) for occurrences of "why," "what for," and "for what." For each novel, the software then produced a text file containing the passages surrounding each occurrence of a term (from 125 characters before to 330 characters after the term). Then the three text files were formatted and printed as separate booklets and handed to two coders.

Coding. The three resulting booklets contained a total of 236 passages ( 70 in MD, 80 in TL, and 86 in PA). Of those, 129 were uncodable and had to be excluded (see the Appendix). The majority of excluded statements were not wonderings (e.g., "Why, it was all damp in here'), concerned nonbehavioral events, or were normative ("Why should she suffer?"). The remaining exclusions followed the criteria discussed in the Appendix (e.g., passive voice, "we" statements). After these and 9 more exclusions of events on which coders could not agree (even after

\footnotetext{
${ }^{2}$ One of the novels, along with numerous others not coded here, is available on the Internet as well (see http://www-cgi.cs.cmu.edu/web/ books.html).
} 
Table 5

Wonderings About Behavioral Events in Study 5 (Novels by Woolf and Joyce)

\begin{tabular}{lccccccc}
\hline & \multicolumn{3}{c}{ Actor } & & \multicolumn{3}{c}{ Observer } \\
\cline { 2 - 3 } \multicolumn{1}{c}{ Behavior } & Intentional & Unintentional & Total & & Intentional & Unintentional & Total \\
\hline Observable & 7 & 1 & 8 & 56 & 3 & 59 \\
Unobservable & 1 & 22 & 23 & & 0 & 8 & 8 \\
Totals & 8 & 23 & & & 56 & 11 & \\
\hline
\end{tabular}

discussion ), 98 wonderings remained: 31 from the actor perspective and 67 from the observer perspective.

Literature poses a unique problem for the coding of perspective because authors often express an actor's wonderings in the grammatical third person (i.e., from the omniscient narrator's perspective): "Mrs. Ramsay, leaving the argument entirely in the hands of the two men, wondered why she was so bored by this talk." (Woolf, 1927. p. 143). Despite their third-person form, these wonderings belong psychologically in the actor category. By contrast, reports of wonderings as entertained by one character about another character belong psychologically in the observer category: "Why, thought Mr. Ramsay, should she look at the sea when I am here?" (Woolf, 1927, p. 225).

After attending a training session based on the written coding instruction ( see the Appendix) and discussions of the unique features of coding novels, each coder worked independently on one booklet at a time. Initial agreement on codability was $84 \%$ ( $\mathrm{MD}=86 \%$, $\mathrm{TL}=84 \%$, and $\mathrm{PA}$ $=83 \%$ ). Among the events both found codable, initial agreernent on perspective was $93 \%(\mathrm{MD}=92 \%, \mathrm{TL}=97 \%$, and $\mathrm{PA}=91 \%)$ and initial agreement on behavior type was $85 \%$ (MD $=81 \%$, TL $=94 \%$, and $\mathrm{PA}=81 \%$ ). Agreement on observability was higher $(95 \%)$ than agreement on intentionality $(90 \%)$. After discussing all the cases in which they had disagreed, the coders' final agreement was as follows: codability, $98 \%$; perspective, $100 \%$; and behavior type, $96 \%$. Only the 98 events on which both coders agreed were used for analysis.

\section{Results and Discussion}

Table 5 shows the distribution of 98 wonderings across the four behavior types for actors and observers. The data were subjected to a three-way (Perspective $\times$ Intentionality $\times$ Observability) $\log$-linear analysis.

The results support both parts of Hypothesis 1: Actors wondered more often about unintentional $(74 \%)$ than intentional ( $26 \%$ ) behaviors, whereas observers wondered more often about intentional $(84 \%)$ than unintentional $(16 \%)$ behaviors. These interactions did not reach significance in a partial test, whereas they were highly significant when using the corresponding marginal test, $\chi^{2}(1)=31.0, p<.001 .^{3}$ Moreover, actors wondered more often about unobservable (74\%) than observable (26\%) behaviors, whereas observers wondered more often about observable $(88 \%)$ than unobservable $(12 \%)$ behaviors. This time, both statistical tests yielded significance: partial $\chi^{2}(1)=6.7$, $p<.01$; marginal $\chi^{2}(1)=38.0, p<.001$.

The partial test for the Perspective $\times$ Intentionality interaction mentioned earlier was not significant because the Intentionality $\times$ Observability interaction was overwhelmingly strong: Only 5 of 98 wonderings were not about actions or experiences, partial $\chi^{2}(1)=56.2, p<.001$ (effect size $r=.76$ ). The Perspective $x$ Intentionality interaction was sizable too (see Table 5), but it was still the weakest effect in the model. Because partial tests adjust the weakest effect for all other effects in the model, they left little power (given the limited sample) for testing the Perspective $\times$ Intentionality effect. Even though the frequency distribution and the corresponding marginal chi-square clearly supported Hypothesis 1, we performed a final test of this hypothesis that held constant the overwhelming Intentionality $\times \mathrm{Ob}$ servability interaction. This was done by analyzing only the 93 actions and experiences.

Considered jointly, the two parts of Hypothesis 1 predicted that actors wonder more often about unintentional and unobservable events (i.e., experiences) than about intentional and observable events (i.e., actions), whereas observers wonder more often about intentional and observable events (actions) than about unintentional and unobservable events (experiences). A twoway $\log$-linear analysis on perspective (actor vs. observer) and behavior type (action vs. experience) supported this joint hypothesis. Actors wondered more about experiences (76\%) than actions ( $24 \%$ ), whereas observers wondered more about actions $(88 \%)$ than experiences $(12 \%)$, partial $\chi^{2}(1)=36.7, p<.001$ (effect size $r=.63$ ). From these and prior analyses, we can conclude that the principles that guide which behaviors people explain apply to works of literature as well as to real life.

\section{Summary}

Figure 2 shows the results of Studies $1-5$. Wonderings (see Figure 2, top) clearly showed the basic actor-observer asymmetry predicted in Hypothesis 1: Actors wondered about few intentional $(27 \%)$ and few observable $(33 \%)$ behaviors, whereas observers wondered about many intentional $(67 \%)$ and many observable $(74 \%)$ behaviors. Private explanations (see Figure 2 , middle) showed the same asymmetry (with virtually identical numbers), as predicted in Hypothesis 2. In communicative explanations, however, this basic actor-observer asymmetry was eliminated (see Figure 2, bottom). In communicative explanations, actors did not answer their own wonderings but clarified their partners' potential wonderings, thus assisting in the

\footnotetext{
${ }^{3}$ In higher order log-linear models, some interactions (here, two-way interactions) can be tested either by a marginal test, which collapses across the variables not included in the interaction, or a partial test, which adjusts the tested interaction for all other effects in the model (e.g., Tabachnik \& Fidell, 1996, pp. 248-249). Partial tests are more conservative, particularly if the model contains strong effects that "consume" most of the variance. This was the case in our analysis, so we report results of hoth tests.
} 


\section{Wonderings}

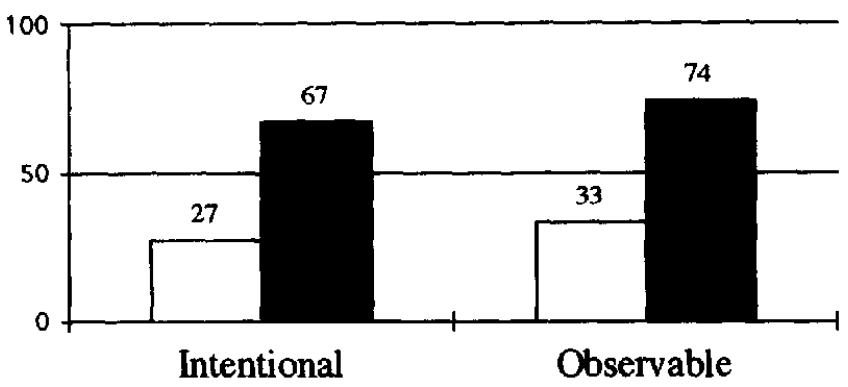

\section{Private explanations}

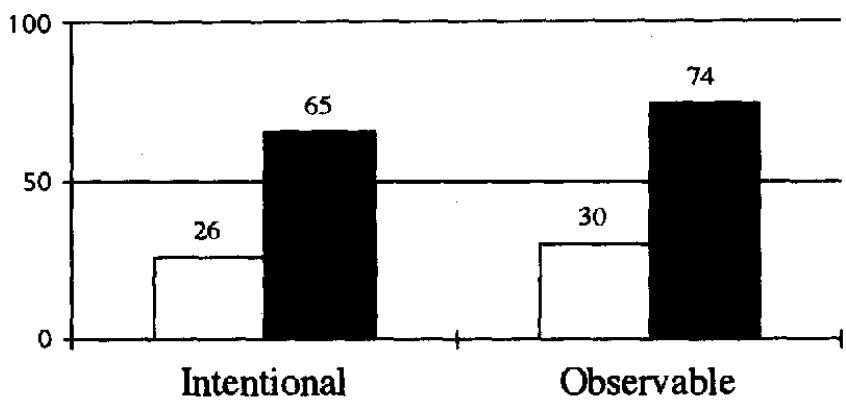

3. Communicative explanations

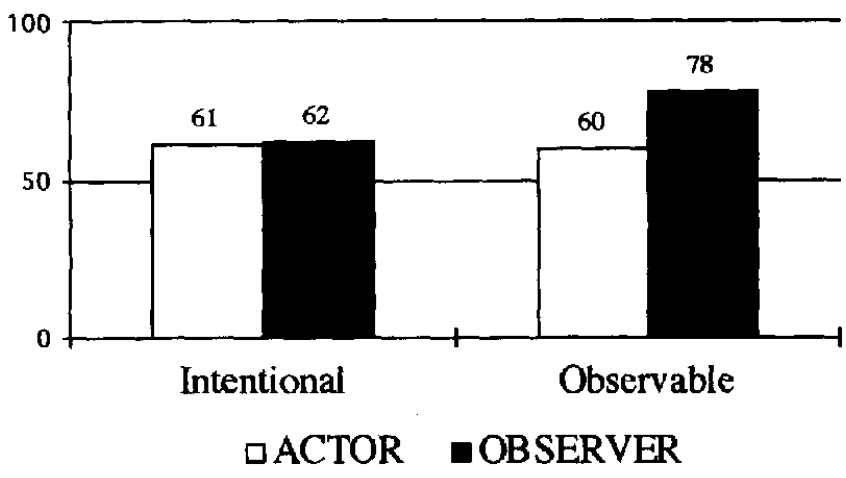

Figure 2. A basic actor-observer asymmetry and its boundary condition. Shown are the percentages of intentional (vs. unintentional) and observable (vs. unobservable) behaviors in wonderings (top), private explanations (middle), and communicative explanations (bottom), aggregated across the five studies.

grounding of interpersonal behavior. That is, actors now explained more intentional $(61 \%)$ and observable $(60 \%)$ behaviors, resembling observers, who also explained more intentional $(62 \%)$ and observable $(78 \%)$ behaviors.

\section{General Discussion}

Our results confirm the predictions we have derived from a general framework of wonderings and explanations. This frame- work clarifies, then, why we found an actor-observer asymmetry in wonderings. Actors wonder mainly about unobservable and unintentional behaviors because these behaviors satisfy the three conditions of actors' wonderings (awareness, nonunderstanding, and relevance). Observers wonder mainly about observable and intentional behaviors because these behaviors satisfy the corresponding three conditions of observers' wonderings. The framework also clarifies why the same actor-observer asymmetry held for private explanations. These explanations are answers to people's own wonderings and therefore refer to the same behaviors that actors and observers tend to wonder about. Finally, the framework clarifies why no actor-observer asymmetry held for communicative explanations. In these explanations, actors answer their partners' wonderings and thus provide explanations for the intentional and observable behaviors about which their partners as observers wonder.

\section{Classification of Behavioral Events}

Because actions are both intentional and observable and experiences are both unintentional and unobservable, the actor-observer asymmetry that we found can be summarized nicely in terms of actions and experiences alone: Actors wondered more about experiences ( $64 \%$ ) than actions $(24 \%)$, whereas observers wondered more about actions (64\%) than experiences $(23 \%)$. In private explanations, actors explained more experiences $(62 \%)$ than actions (17\%), whereas observers explained more actions $(61 \%)$ than experiences ( $20 \%)$. In communicative explanations, however, actors explained more actions (50\%) than experiences $(28 \%)$, resembling observers, who also explained more actions ( $59 \%$ ) than experiences $(20 \%)$.

In light of this summary, it seems as though we could simply reduce the fourfold classification of behavioral events proposed here to a twofold classification of only actions and experiences. Such a reduction, however, would imply classification problems for the behavioral events that we had labeled mere behaviors and intentional thoughts. For example, would "Dion blushed" be reclassified as an action or an experience? Would "Farah decided to go to the play" be considered an experience or an action? If we were unable to solve these classification problems, we would have to discard about $20 \%$ of people's behavior explanations and wonderings.

Even if we were to exclude all mere behaviors and intentional thoughts, we would still detect a significant actor-observer asymmetry between actions and experiences. However, we would not know why this asymmetry arose. A twofold conceptualization of behavioral events cannot explain the basic actorobserver asymmetry demonstrated in the current studies. Because actions are both intentional and observable and experiences are both unintentional and unobservable, the actor-observer asymmetry between actions and experiences could have been due either to intentionality or to observability. Inclusion of all four behavioral events that result from crossing intentionality and observability resolved this ambiguity because it demonstrated that both intentionality and observability contributed to the actor-observer asymmetry. More specifically, when we applied the general conditions of wondering (awareness, nonunderstanding, and relevance) to the specific case of behavior explanations, we found that awareness explained the contribu- 
tion of observability and that nonunderstanding and relevance explained the contribution of intentionality.

Thus, in light of the general conditions of wondering and explanation, only the fourfold classification of behavioral events helps clarify why actors tend to explain actions and why observers tend to explain experiences. Moreover, the fourfold classification helps clarify why actors and observers tend not to explain intentional thoughts and mere behaviors, as described next.

Actors fail to explain intentional thoughts because these events are intentional (the condition of nonunderstanding is not met). By contrast, observers fail to explain intentional thoughts because these events are unobservable (the condition of awareness is not met). These distinct processes lead to distinct predictions that cannot be derived from a twofold action-experience classification alone. For example, we expect that actors would begin to wonder about their own intentional thoughts if they were long past (satisfying the nonunderstanding condition). Similarly, observers would begin to wonder about actors' intentional thoughts if they gained access to those events (satisfying the awareness condition).

In the same vein, actors fail to explain mere behaviors (e.g., their own facial expressions or gestures) because these events, even though publicly observable, are difficult to notice from their perspective (Storms, 1973); thus, the condition of awareness is not satisfied. By contrast, observers fail to explain mere behaviors because these events are unintentional and are therefore regarded as less diagnostic and consequential, so the condition of relevance is not satisfied. These distinct processes again lead to distinct predictions that cannot be derived from a twofold action-experience classification alone. For example, we expect that actors would begin to wonder about their own gestures if they saw them on video playback (satisfying the awareness condition). Similarly, observers would begin to wonder about actors' gestures if told that these behaviors are highly diagnostic (thus satisfying the relevance condition).

In addition to these distinct processes that tend to prevent actors and observers from explaining mere behaviors and intentional thoughts, there also is a general tendency across actors and observers to explain few mere behaviors and intentional thoughts but many actions and experiences. (This tendency was represented by a consistent Intentionality $\times$ Observability interaction throughout our five studies.) Why this general tendency? Why do people, both actors and observers, wonder about and explain mainly actions and experiences?

The prevalence of actions and experiences may be a result of the very asymmetries we have documented. Because actors care mostly about unintentional and unobservable events (i.e., experiences) and observers care mostly about intentional and observable events (i.e., actions), and because people constantly switch between these two perspectives (at times holding both simultaneously), they naturally consider these two event types more than any other ones. People writing in their diaries are so used to considering actions from the observer perspective that they will also explain some actions from the actor perspective (e.g., "I didn't go to class today because I felt terrible"). Similarly, social perceivers are so used to making sense of emotions from the actor perspective that they will try to make sense of others' emotions from the observer perspective as well (even if they can only infer them). Actors will still explain many more experi- ences than actions, and observers will still explain many more actions than experiences, but summed across perspectives, explanations of actions and experiences are likely to accumulate. - This accumulation also would increase the frequency of using action verbs and experience verbs, explaining why past research reliably identified those verb classes (cf. All, 1986; Brown \& Fish, 1983; McArthur, 1972). Furthermore, the availability of more action and experience verbs in turn facilitates people's thinking and talking about actions and experiences, thus feeding back into the initial prevalence of explaining those events.

The general tendency of people to explain actions and experiences more than any other behavioral event thus may be rooted in the basic actor-observer asymmetry, which itself is caused by the three conditions of wondering that differentially favor actors' and observers' explanations of intentional and observable behaviors. Even the prevalence of actions and experiences must be understood, then, within the entire framework of wondering about and explaining behaviors that differ in observability and intentionality.

\section{Negativity and Unexpectedness Within the Current Framework}

As shown earlier, the conditions for wonderings and explanations (awareness, nonunderstanding, and relevance) provide a framework for understanding why negative and unexpected events trigger attributions (cf. Weiner, 1985). Moreover, negativity and unexpectedness should interact with the basic actorobserver asymmetry demonstrated in our studies. For example, intentional actions will more often be surprising to observers than to actors. Also, when actors give private explanations, they will explain behaviors that they themselves consider negative. However, when they offer communicative explanations, they will explain behaviors that they think their partner considers negative.

\section{Interpersonal Implications of the Basic Actor-Observer Asymmetry}

If actors and observers explain different types of behaviors, we might hypothesize that some interpersonal (e.g., marital) conflicts prove difficult to resolve because the two partners are actually arguing about different behavioral events. For example, one person (the actor) focuses on his or her experience of being angry and hurt, whereas the partner (as observer) focuses on the actor's action of yelling. The conflict may be difficult to resolve as long as the two people continue to talk about two different events (cf. Ross \& Ward, 1995; Tannen, 1990).

Such conflicts can be avoided in one of two ways. First, observers can become more like actors, focusing more on actors' experiences than their actions. Second, actors can become more like observers, focusing more on their own actions than on their own experiences. Interestingly, our data show that this second solution occurs naturally in communicative contexts. When explaining themselves to an observer, actors explain more intentional than unintentional behaviors and more observable than unobservable behaviors. Communication between actors and observers apparently shifts actors' attention away from their own 
unobservable behaviors and may thus reduce interpersonal conflict.

However, the actor's resulting shift away from unobservable behaviors creates a new problem. Studies show that information about unobservable behaviors is particularly diagnostic of an actor's personality (Andersen, 1984). Yet, observers are unlikely to receive this type of information. In communicative contexts-the only context in which observers receive information from the actor at all-actors rarely explain unobservable behaviors and thus deprive observers of the most diagnostic type of information.

Intimacy might help avoid this problem in several ways. First, actors may explain more unobservable behaviors to people with whom they have intimate relationships. If a stranger asks Anne about her day, she will probably explain her observable behaviors ("I worked out for $2 \mathrm{hr}$ this morning because . . ."), but if a friend asks the same question, she may be more willing to explain her unobservable behaviors ("I felt so great during my workout this morning because....').

Second, observers may wonder about the unobservable behaviors of those with whom they have intimate relationships. In particular, intimate observers may feel comfortable asking their partners to explain some of their thoughts and feelings to them. Third, observers who are talking about a third person may explain the person's unobservable behaviors if they are on intimate terms with that person. If Anne is talking about President Clinton, she is unlikely to explain his feelings, thoughts, or desires. However, if the conversation shifts to her best friend Ben, she may provide many explanations for Ben's unobservable behaviors. Fourth, observers may explain a third person's unobservable behaviors to a conversation partner if they are on intimate terms with that conversation partner. In the presence of her most intimate friends, for example, Anne feels comfortable explaining Ben's thoughts and feelings. When speaking with a stranger, however, she confines herself to explaining his observable behaviors.

In summary, we expect that actors in intimate conversations return to their normal pattern of explaining their own unobservable behaviors, and we also expect that observers wonder about and explain more unobservable behaviors if they are intimate either with the person about whom they talk or the conversation partner with whom they speak. These hypotheses, of course, must be tested.

\section{Theoretical Implications of the Basic Actor-Observer Asymmetry}

The regularities that dictate which behaviors people explain also might influence how they explain them. That is, actors' and observers' differential interest in explaining certain behaviors might have consequences for the kinds of explanations they provide. Further study of these consequences might shed light on a number of issues in attribution research (Malle, 1996).

Questionnaire assessments of attributional styles (e.g., Fincham, Beach, \& Baucom, 1987; Fincham \& Bradbury, 1992; Seligman, Abramson, Semmel, \& Von Baeyer, 1979) are often limited to behavioral events selected by the experimenters, not by the participants themselves. Participants are asked to rate the behaviors (typically observable events) along several attribu- tional dimensions. The resulting assessments surely apply to the selected behaviors, but do they also apply to the behaviors people themselves are interested in explaining?

One might suspect that the predictive power of such attributional style measures could be enhanced by taking into account which behavioral events people themselves tend to explain. This approach calls for the study of naturally occurring explanations. One would need to examine an entire stream of behaviors, select the behavioral events that people care to explain, and examine their different explanations for those events. To our knowledge, only a few researchers have attempted to code naturally occurring explanations (e.g., Holtzworth-Munroe \& Jacobson, 1988), with only limited success. Perhaps greater success could be achieved by first sorting behavioral events into distinct types (e.g., observable and unobservable, intentional and unintentional) and then separately examining the explanations within each type (cf. Bradbury \& Fincham, 1988). The results would then indicate whether, say, quarreling partners differ in their explanations for all behavior types or only for certain diagnostic types.

The distinction between different types of behaviors might also shed new light on the classic actor-observer asymmetry, the thesis that actors tend to explain their own behaviors with situational causes, whereas observers tend to explain others' behaviors with person causes (Jones \& Nisbett, 1972). Many attribution theorists (e.g., Heider, 1958; Jones \& Nisbett, 1972) explained this asymmetry in terms of differences in attention: Because the observer attends to the actor and the actor attends to the surrounding situation, observers provide actor-centered explanations, whereas observers provide situation-centered explanations. Put simply, "what you attend to is what you attribute to" (Ross \& Nisbett, 1991, p. 140).

However, this claim leads to a puzzle. When observers attend to the actor's actions, they give more person explanations, as highlighted in salience studies (e.g., McArthur \& Ginsberg, 1981; Robinson \& McArthur, 1982; Taylor \& Fiske, 1978), yet, when they attend to the actor's experiences, they give more situational explanations, as highlighted in empathy studies (Galper, 1976; Gould \& Sigall, 1977; Regan \& Totten, 1975; Wegner \& Finstuen, 1977). Contradicting the classic attentionattribution thesis, these two groups of observers attended to the same "object" (the actor) but provided different types of explanation.

To solve this puzzle, we need to distinguish between different types of behavior and identify the characteristic explanations they elicit. The results of several studies suggest that people tend to explain experiences with characteristics of the situation and actions with characteristics of the person (e.g., Au, 1986; Brown \& Fish, 1983; Gilovich \& Regan, 1986; Karasawa, 1995; Kruglanski, 1975). Therefore, by putting the focus on the actor's experiences, empathy studies invited more situation attributions; conversely, by putting the focus on the actor's actions, salience studies invited more person attributions. The attentionattribution puzzle thus can be resolved by considering that attention influences attribution via attention to different behavior types (even of the same person), which in turn elicit different attributions.

This link between behavior types and explanations might also account for some of the classic actor-observer findings. If actors 
are more likely to explain experiences, as demonstrated in this article, and if experiences are more likely to be explained by situational causes, actors are overall more likely to provide situational explanations. By contrast, if observers are more likely to explain actions and if actions are usually explained by person causes, observers are overall more likely to provide person explanations. As a result, actors would seem to provide more situational explanations, whereas observers would seem to provide more person explanations. However, such a difference would rest on actors' and observers' tendency to explain different behaviors rather than on their tendency to explain the same behaviors with different causes.

This analysis applies to a class of actor-observer studies in which people provided explanations for entire interactionsan undefined set of behavioral events-rather than for clearly identified behavioral events (e.g., Arkin \& Duval, 1975; Moore, Sherrod, Liu, \& Underwood, 1979; Robins, Spranca, \& Mendelsohn, 1996; Storms, 1973). To the extent that such interactions contained both actions and experiences, the actor-observer asymmetry found in these studies may be spurious because actors may have focused on their experiences (and explained them with situation factors), whereas observers may have focused on the actor's actions (and explained them with person factors ). The overall attributions with respect to the entire interaction are therefore equivocal because actors and observers may have based them on different types of behaviors. Future research must test this hypothesis.

\section{Conclusion}

We have examined which different behaviors actors and observers wonder about and explain. We first presented a framework of psychological conditions for wondering why and explaining why particular events occur. We then applied this framework to behavioral events, revealing a basic actor-observer asymmetry with a broad range of implications. The asymmetry provides insight into certain interpersonal conflicts and offers new methods of overcoming these conflicts. Furthermore, it highlights the impact of the communicative context on people's explanations, thus challenging the assumption that the process of attribution is entirely cognitive (cf. Antaki, 1988; Draper, 1988; Hilton, 1990). Finally, the basic actor-observer asymmetry challenges another assumption of classic attribution research: that all behaviors are alike and that behavior explanations therefore differ only in the causes they mention. Because not all behaviors are alike, people's tendencies to explain certain types of behaviors may strongly influence the kinds of explanations they will give. Recognizing this influence sheds new light on several issues in attribution theory, such as the study of individual differences in explanatory style, the attention-attribution link, and the classic actor-observer asymmetry. We hope that future researchers will consider carefully the implications of this basic asymmetry and explore jointly which behaviors people explain and how they explain them.

\section{References}

Andersen, S. M. (1984). Self-knowledge and social inference: II. The diagnosticity of cognitive/affective and behavioral data. Journal of Personality and Social Psychology, 46, 294-307.
Antaki, C. (1988). Explanations, communication and social cognition. In C. Antaki (Ed.), Analyzing everyday explanation: A casebook of methods (pp. 1-14). London: Sage.

Arkin, R. M., \& Duval, S. (1975). Focus of attention and causal attributions of actors and observers. Journal of Experimental Social Psychology, 11, 427-438.

An, T, K. (1986). A verb is worth a thousand words: The causes and consequences of interpersonal events implicit in language. Journal of Memory and Language, 25, 104-122.

Belmore, S. M. (1987). Determinants of attention during impression formation. Journal of Experimental Psychology: Learning, Memory, and Cognition, 13, 480-489.

Berlyne, D. E. (1960). Conflict, arousal, and curiosity. New York: McGraw-Hill.

Bohner, G., Bless, H., Schwarz, N., \& Strack, F. (1988). What triggers causal attributions? The impact of valence and subjective probability. European Journal of Social Psychology, 18, 335-345.

Boucher, J., \& Osgood, E. E. (1969). The Pollyanna hypothesis. Journal of Verbal Learning and Verbal Behavior, 8, 1-8.

Bradbury, T. N., \& Fincham, F. D. (1988). Assessing spontaneous attributions in marital interaction: Methodological and conceptual considerations. Journal of Social and Clinical Psychology, 7, 122-130.

Bromberger. S. (1965). An approach to explanation. In R. Butler (Ed.), Analytical philosophy (2nd series, pp. 72-105). Oxford, England: Basil Blackwell.

Brown, R., \& Fish, D. (1983). The psychological causality implicit in language. Cognition, 14, 237-273.

Buss, A. R. (1978). Causes and reasons in attribution theory: A conceptual critique. Journal of Personality and Social Psychology, 36, 1311 1321.

Buss, D. M., \& Craik, K. H. (1983). The act frequency approach to personality. Psychological Review, 90, 105-126.

Clark, H. H., \& Brennan, S. E. (1991). Grounding in communication. In L. B. Resnick, J. M. Levine, \& S. D. Teasley (Eds.), Socially shared cognition (pp. 127-149). Washington, DC: American Psychological Association.

Clark, H. H., \& Schaefer, E. F. ( 1989). Contributing to discourse. Cognitive Science, 13, 259-294.

Draper, S. W. (1988). What's going on in everyday explanations? In C. Antaki (Ed.), Analyzing everyday explanation: A casebook of methods (pp. 15-31). London: Sage.

Festinger, L. (1957). A theory of cognitive dissonance. Stanford, CA: Stanford University Press.

Fiedler, K., \& Semin, G. (1988). On the causal information conveyed by different interpersonal verbs: The role of implicit sentence context. Social Cognition, 6, 21-39.

Fincham, F. D., Beach, S. R., \& Baucom, D. H. (1987). Attribution processes in distressed and nondistressed couples: 4 . Self-partner attribution differences. Journal of Personality and Social Psychology, 52, $739-748$.

Fincham, F. D., \& Bradbury, T. N. (1992). Assessing attributions in marriage: The relationship attribution measure. Journal of Personality and Social Psychology, 62, 457-468.

Fiske, S. T., \& Taylor, S. E. (1991). Social cognition (2nd ed.). New York: McGraw-Hill.

Funder, D. C. (1980). On seeing ourselves as others see us: Self-other agreement and discrepancy in personality ratings. Journal of Personality, 48, 473-493.

Funder, D. C., \& Dobroth, K. M. (1987). Differences between traits: Properties associated with interjudge agreement. Journal of Personality and Social Psychology, 52, 409-418.

Galper, R. E. (1976). Turning observers into actors: Differential causal attribution as a function of "empathy." Journal of Research in Personality, $10,328-335$. 
Gilovich, T., \& Regan, D. T. (1986). The actor and the experiencer: Divergent patterns of causal attribution. Social Cognition, 4, 342352.

Gould, R., \& Sigall, H. (1977). The effects of empathy and outcome on attribution: An examination of the divergent-perspective hypothesis. Journal of Experimental Social Psychology, 13, 480-491.

Hastie, R. (1984). Causes and effects of causal attribution. Journal of Personality and Social Psychology, 46, 44-56.

Heider, F. (1958). The psychology of interpersonal relations. New York: Wiley.

Hilton, D. J. (1990). Conversational processes and causal explanation. Psychological Bulletin, 107, 65-81.

Hilton, D. J., \& Slugoski, B. R. (1986). Knowledge-based causal attribution: The abnormal conditions focus model. Psychological Review, $93,75-88$.

Holtzworth-Munroe, A., \& Jacobson, N. S. (1988). Toward a methodology for coding spontaneous causal attributions: Preliminary results with married couples. Journal of Social and Clinical Psychology, 7, $101-112$.

Janoff-Bulman, R. (1992). Shattered assumptions: Towards a new psychology of trauma. New York: Free Press.

John, O. P., \& Robins, R. W. (1993). Determinants of interjudge agreement on personality traits: The Big Five domains, observability, evaluativeness, and the unique perspective of the self. Journal of Personality, 61, 521-551.

Jones, E. E., \& Davis, K. E. (1965). From acts to dispositions: The attribution process in person perception. Advances in Experimental Social Psychology, 2, 219-266.

Jones, E. E., \& Nisbett, R. E. (1972). The actor and the observer: Divergent perceptions of the causes of behavior. In E. E. Jones, D. Kanouse, H. H. Kelley, R. E. Nisbett, S. Valins, \& B. Weiner (Eds.), Attribution: Perceiving the causes of behavior (pp. 79-94). New York: General Learning Press.

Jones, E. E., \& Thibaut, J. W. (1958). Interaction goals as bases of inference in interpersonal perception. In $R$. Tagiuri \& $\mathrm{L}$. Petrullo (Eds.), Person perception and interpersonal behavior (pp. 151178). Stanford, CA: Stanford University Press.

Joyce, J. (1993). A portrait of the artist as a young man. New York: Garland. (Original work published 1916)

Karasawa, K. (1995). An attributional analysis of reactions to negative emotions. Personality and Social Psychology Bulletin, 21, 456-467.

Kelley, H. H. (1967). Attribution theory in social psychology. In D. Levine (Ed.), Nebraska Symposium on Motivation (Vol. 15, pp. 129 240). Lincoln: University of Nebraska Press.

Kenrick, D. T., \& Stringfield, D. O. (1980). Personality traits and the eye of the beholder: Crossing some traditional philosophical boundaries in the search for consistency in all of the people. Psychological Review, $87,88-104$.

Kiely, R. (1980). Beyond egotism: The fiction of James Joyce, Virginia Woolf, and D. H. Lawrence. Cambridge, MA: Harvard University Press.

Kruglanski, A. H. (1975). The endogenous-exogenous partition in attribution theory. Psychological Review, 82, 387-406.

Kruglanski, A. H. (1989). Lay epistemics and human knowledge. New York: Plenum.

Locke, D., \& Pennington, D. (1982). Reasons and other causes: Their role in attribution processes. Journal of Personality and Social Psychology, 42, 212-223.

Malle, B. F. (1996). Intentionality in attribution: A theory of the folk explanations of behavior. Manuscript submitted for publication.

Malle, B. F., \& Knobe, J. (in press). The folk concept of intentionality. Journal of Experimental Social Psychology.

McArthur, L. Z. (1972). The how and what of why: Some determinants and consequences of causal attribution. Journal of Personality and Social Psychology, 22, 171-193.

McArthur, L. Z., \& Ginsberg, E. (1981). Causal attribution to salient stimuli: An investigation on visual fixation mediators. Personality and Social Psychology Bulletin, 7, 547-553.

Miller, G. R., \& Steinberg, M. (1975). Between people: A new analysis of interpersonal communication. Chicago: Science Research Associates.

Moore, B. S., Sherrod, D. R., Liu, T. J., \& Underwood, B. (1979). The dispositional shift in attribution over time. Journal of Personality and Social Psychology, 15, 553-569.

Pepitone, A. (1968). The problem of motivation in consistency models. In R. P. Abelson, E. Aronson, W. J. McGuire, T. M. Newcomb, M. J. Rosenberg, \& P. H. Tennenbaum (Eds.), Theories of cognitive consistency: A sourcebook (pp. 319-326). Chicago: Rand McNally.

Pratto, F., \& John, O. P. (1991). Automatic vigilance: The attentiongrabbing power of negative social information. Joumal of Personality and Social Psychology, 61, 380-391.

Pyszczynski, T. A., \& Greenberg, J. (1981 ). Role of disconfirmed expectancies in the instigation of attributional processing. Joumal of Personality and Social Psychology, 40, 31-38.

Read, S. J., \& Miller, L. C. (1994). Dissonance and balance in belief systems: The promise of parallel constraint satisfaction processes and connectionist modeling approaches. In R. C. Schank \& E. Langer (Eds.), Beliefs, reasoning, and decision making: Psycho-logic in honor of Bob Abelson (pp. 209-235). Hillsdale, NJ: Erlbaum.

Regan, D. T., \& Totten, J. (1975). Empathy and attribution: Turning observers into actors. Journal of Personality and Social Psychology, $32,850-856$.

Robins, R. W., Spranca, M. D., \& Mendelsohn, G. A. (1996). The actorobserver effect revisited: Effects of individual differences and repeated social interactions on actor and observer attributions. Journal of Personality and Social Psychology, 71, 375-389.

Robinson, J., \& McArthur, L. Z. ( 1982). Impact of salient vocal qualities on causal attribution for a speaker's behavior. Journal of Personality and Social Psychology, 43, 236-247.

Ross, L., \& Nisbett, R. E. (1991). The person and the situation. New York: McGraw-Hill.

Ross, L., \& Ward, A. (1995). Naive realism in everyday life: Implications for social conflict and misunderstanding. In T. Brown, E. Reed, \& E. Turiel (Eds.), Values and knowledge (pp. 103-135). Hillsdale, NJ: Eribaum.

Ross, M., \& Fletcher, G. J. O. (1985). Attribution and social perception. In G. Lindzey \& E. Aronson (Eds.), Handbook of social psychology (pp. 73-122). New York: Random House.

Scott, M. B., \& Lyman, S. M. (1968). Accounts. American Sociological Review, 33, 46-62.

Seligman, M. E. P., Abramson, L. Y., Semmel, A., \& Von Baeyer, C. (1979). Depressive attributional style. Journal of Abnormal Psychology, 88, 242-247.

Shaver, K. G. (1985). The attribution of blame. New York: SpringerVerlag.

Steele, C. M. (1988). The psychology of self-affirmation: Sustaining the integrity of the self. Advances in Experimental Social Psychology, $21,261-302$.

Storms, M. D. (1973). Videotape and the attribution process: Reversing actors' and observers' point of view. Journal of Personality and Social Psychology, 27, 165-175.

Svartvik, J. (Ed.). (1990). The London-Lund corpus of spoken English: Description and research. Lund, Sweden: Lund University Press.

Svartvik, J., \& Quirk, R. (Eds.). ( 1980). A corpus of English conversation (Lund Studies in English, 56). Lund, Sweden: Gleerups/Liber.

Tabachnik, B. G., \& Fidell, L. S. (1996). Using multivariate statistics (3rd ed.). New York: HarperCollins. 
Tannen, D. (1990). You just don't understand. New York: Ballantine Books.

Taylor, S. E. (1983). Adjustment to threatening events: A theory of cognitive adaption. American Psychologist, 38, 1161-1173.

Taylor, S.E. (1991), Asymmetrical effects of positive and negative events: The mobilization-minimization hypothesis. Psychological Bulletin, 110, 67-85.

Taylor, S. E., \& Fiske, S. T. (1978). Salience, attention, and attribution: Top of the head phenomena. Advances in Experimental Social Psychology, 11, 249-288.

Watson, D. (1982). The actor and the observer: How are their perceptions of causality divergent? Psychological Bulletin, 92, 682-700.

Wegner, D. M., \& Finstuen, K. (1977). Observers' focus of attention in the simulation of self-perception. Journal of Personality and Social Psychology, 35, 56-62.

Weiner, B. (1985). "Spontaneous" causal thinking. Psychological Bulletin, 97, 74-84.

White, P. A. (1991). Ambiguity in the internal/external distinction in causal attribution. Journal of Experimental Social Psychology, 27. 259-270.

Wong, P. T., \& Weiner, B. (1981). When people ask "why" questions, and the heuristics of attributional search. Journal of Personality and Social Psychology, 40, 246-259.

Woolf, V. (1927). To the lighthouse. New York: Harcourt, Brace. Woolf, V. (1925). Mrs. Dalloway. New York: Harcourt, Brace.

\section{Appendix}

\section{Coding Scheme for Behavioral Events}

This coding system ${ }^{\mathrm{A}}$ is an attempt to classify the types of behaviors people wonder about and explain. Our interest is limited to behavioral and psychological events (i.e., actions, thoughts, feelings). We thus exclude explanations of inanimate mechanisms (e.g., explaining how a diesel engine works), global facts (e.g., explaining why there is so much conflict in the world), and nonpsychological facts (e.g., explaining why Jean has deep blue eyes). In short, we focus on explanations of why a person is $x^{\prime}$ ing, where $x$ stands for an action, a feeling, or any other behavioral or psychological event.

We code two categories of events: (a) When people explain a behavior, we call this behavior the object of explanation. For example, in the statement "I am sad because he doesn't call me anymore," the object of explanation is her state of sadness. In "Sharon relaxed around my family because they are cool people," the object of explanation is Sharon's relaxing. (b) When people wonder about a behavior, we call this behavior the object of wondering. Wonderings are "why" questions that may or may not be followed by an actual explanation (e.g., "Why is he so grumpy today?"'). For both categories of events (i.e., objects of explanations or objects of wondering), we ask the following four questions: 1 . Is the explanation private or communicative? 2 . Is the agent of the explained behavior or experience self or other? 3. Was the explained event observable or unobservable? 4 . Was the explained event intentional or unintentional?

\section{Question 1: Is the Explanation Private or Communicative?}

Private explanations are directed to oneself. That is, a person explains an event to herself because she is wondering why the event occurred (such as when writing in a diary or in "self-talk"). Communicative explanations are directed to other people. That is, the speaker explains an event to another person because he believes that the other person is wondering why the event occurred (such as in conversations or letters). In some studies, participants are asked to describe the circumstances under which the explanation or wondering took place (e.g., self-talk or conversation), so we can often derive the type of explanation (private or communicative) from this circumstance information. In other studies, we have made more indirect inferences. Occasionally, participants give direct clues, such as "My boss asked me why I didn't . . ." or "I explained to my mother why she. . ." Other times, the clues are less direct, such as that the explanation is marked as a direct quote apparently addressed to another person.

\section{Question 2: Is the Agent the Self or Other?}

We must also determine whether a person's explanation or wondering is about herself (self) or about somebody else (other). (Self is often labeled actor, whereas other is labeled observer.) Clear contrast cases are "I was frustrated with school because . . ." ( self) and "My friend was late because. . .' (other). The grammatical subject of the behavior description typically indicates the agent of the explained event: Firstperson propositions indicate self ("I was frustrated . . ."), and secondand third-person propositions indicate other ("My friend was late . . ."). Exceptions include statements in the passive voice (which are uncodable) or propositions such as "My paper was late because . . .," which, on the surface, refers to a paper but implicitly may describe somebody's action. In this particular case, the context allowed a synonymous reformulation (e.g., "Why I turned in my paper late") that helped identify the agent (in this case, the self).

Note that the agent code is independent of the type of explanation (private vs. communicative, Question 1). I might explain to myself either my own behavior or someone else's hehavior; likewise, I might explain to my conversation partner either my own behavior or someone else's behavior.

\section{Question 3: Was the Explained Event Observable or Unobservable?}

Psychologists traditionally have distinguished between publicly observable events (e.g., behaviors, actions) and publicly unobservable events (e.g., bodily sensations, feelings, and thoughts). An event is publicly unobservable when only the agent himself or herself can, in principle, perceive it (e.g., "Why was I so frustrated with my parents?").

Note that many emotions, although primarily unobservable, have observable "symptoms." The frustrated person in the previous example might have subsequently displayed her frustration through (observable) facial expressions; the original event, however, remains unobservable. She may have shown observable symptoms of frustration, but such symptoms were not the object of the wondering; rather, she was trying to explain the frustration itself. Another example of the same problem is, "Why was the professor so insecure when talking to a student after

\footnotetext{
${ }^{\text {Al }}$ Future updates of this coding scheme can be downloaded from the World Wide Web at /http://darkwing.uoregon.edu/ interact/ bev.html $>$.
} 
class?" The speaker may have noted signs of insecurity and therefore wondered about it; the object of wondering, however, is not these signs but the insecurity itself (an unobservable event). Similarly, "being upset" is usually coded as an internal event both from the actor's and the observer's viewpoint, even though an observer uses observable clues to infer this event, whereas the actor often simply feels it. (Occasionally, people refer to behavioral events when they say that someone "got upset." The context may clarify whether the speaker is really referring to an observable event or is using an observable symptom to infer the agent's unobservable emotional state.) Further examples of unobservable events include the following: "I wondered why I wanted to go dancing." "why I didn't like her to sit beside me," and "why my friend was angry at me." Further observable events include the following: "I wondered why I did poorly on the midterm," "why my boyfriend didn't say hello to my best friend," and "why he constantly switched topics."

These examples also illustrate that nonactions (e.g., "He didn't say hello because . . .") do not pose a particular coding problem. Nonactions are observable if we can observe that the action did not occur.

More problematic cases are propositions containing the verbs to decide or to try because they can be either observable or unobservable depending on context. For example, "I wonder why he decided to leave early last night" refers to an observable event, even though the speaker uses the verb decided. True, the decision itself is unobservable, but the speaker seems to be wondering not about the person's mental act of deciding but about his observable behavior of leaving so early. (The use of the vert decide serves to indicate the intentionality of the behavior.) By contrast, "Why I decided not to visit my girlfriend next weekend" refers to the mental act of deciding (especially given that the action has not yet been taken) and must therefore be considered unobservable.

Similarly, the word trying sometimes refers to observable events, sometimes to unobservable ones. "Why he tried to suppress his anger" refers to an unobservable attempt at emotional self-regulation. By contrast, "Why I was trying to cut thicker slices" refers to an observable attempt to cut the bread a certain way; in this case, the word trying seems to indicate that the actor intentionally cut thicker slices. As a rule, if the proposition refers to the mental act of trying, the event is unobservable; if the proposition simply means that an observable behavior was done intentionally, the event is observable.

\section{Question 4: Was the Explained Event Intentional?}

An action is intentional if the agent chose to do it. For example, an explanation of "why my boyfriend didn't say hello to my best friend" refers to an intentional event; apparently, the person chose not to say hello. By contrast, an explanation of "why she was blushing" refers to an unintentional event because the agent apparently did not choose to blush.

Although people show considerable agreement in their judgments of intentionality (we found alpha reliabilities of .99 ), there are a few ambiguous cases that we must clarify. First, choosing to do something does not require that the action be entirely attractive. Paying taxes is not enjoyable, but when I file my tax return $I$ do it intentionally because I would rather pay taxes than risk paying possible fines. Similarly, "I wonder why my friend left without telling anyone" refers to an intentional act, even though the friend might have run to help someone in an emergency; he chose to help immediately rather than informing other people first. In short, actions that meet an obligation, even if the agent meets it unhappily, are intentional actions.

Second, a given event could be intentional in some contexts and unintentional in others, so we must always examine the context (e.g., the explanation of the event). The event expressed in "why Bob is extremely annoying to some women" could be intentional, but the explanation "he means well but doesn't really have the social skills" suggests that Bob is not intentionally annoying-he just comes across that way.
Third, the words can and could are ambiguous. For example, the proposition "why I couldn't go to the party" may refer to a true inability or to a decision under strong pressure. For example, given the explanation "because my mom was visiting," we can assume that the person chose to stay home. In this case, she was not truly incapable of going to the party but decided against it. By contrast, given the explanation "I broke my leg," we must conclude that the person was physically incapable of going to the party. Her behavior was therefore unintentional.

Fourth, habitual behaviors also can be ambiguous. We must first ascertain whether an event describes a disposition (uncodable) or a recurring behavior (codable). If it is a behavioral tendency, we must determine the intentionality of this recurring behavior. For example, does Anne's wondering "why she procrastinates so much" refer to an intentional habit? Even though some of the individual cases might be intentional (e.g., once Anne intentionally procrastinated on a paper because she was waiting for a good idea), the habit itself is clearly unintentional. Anne never decided "to procrastinate so much."

\section{Putting It Together: Four Types of Events}

The observability of a behavioral event and its intentionality are orthogonal and thus combine to produce four $(2 \times 2)$ types of behaviors (see Table Al).

Behavior Type 1 is observable and intentional. Examples include "My friend left without telling anyone," "She has been ignoring me," "I was fighting with a fellow student," and "I am not taking organic chemistry."

Behavior Type 2 is observable and unintentional. Examples include "My brother sometimes has muscle spasms before he falls asleep" and "I stayed up late because my watch stopped running."

Behavior Type 3 is intentional and unobservable. Examples include "He decided to come up with another word," "She is trying to imagine a green meadow," and "I was seriously considering psychology as my major." This type also includes formulations of plans or intentions, such as "Why did he decide not to see his girlfriend next weekend?" and "I want to write to mom and dad (Why? To tell them that I miss them)."

Note that even though the last example contains the word want, it must be considered an intention (Type 3) rather than a mere desire (Type 4). If a person has considered reasons for wanting to do something and is adopting a plan to do it, the word want refers to an intention (Type 3 ). If the person simply wants something, and this wanting emerged spontaneously rather than being adopted through deliberation, want refers to a mere desire (Type 4).

Behavior Type 4 is unintentional and unobservable. These events include bodily states ("Why am I feeling so tired today?"), sensations ("My arm feels numb"), emotions and feelings ("I became frustrated with my parents"), and mere desires ("I wish he would stop; it hurts").

\section{Uncodable Statements}

Some of the passages that contain a linguistic marker for an explanation (e.g., "because") or a wondering (e.g., "why") are uncodable. One general reason is that the event explained is not a behavioral or psychological event but a mechanical event ("My watch had stopped because .. .") or a world event ("The concert didn't start on time

Table Al

Classification of Behavioral Events

\begin{tabular}{lll}
\hline \multicolumn{1}{c}{ Behavior } & \multicolumn{1}{c}{ Intentional } & \multicolumn{1}{c}{ Unintentional } \\
\hline Observable & 1 (Actions) & 2 (Mere behaviors) \\
Unobservable & 3 (Intentional thoughts) & 4 (Experiences) \\
\hline
\end{tabular}


because . . ."). Another general reason is that the wondering is not a wondering why but a wondering if, or the explanation is not an explanation why but an explanation how or explanation that. More specific classes of uncodable statements are discussed below.

\section{Ambiguous Agent}

Statements in the first-person plural (e.g., "why we broke up") are uncodable because we do not know whose behavior is explained-the actor's, the observer's, or both.

Statements in the passive voice ("Why was he sent to that place?") are uncodable for the same reason. The passive voice leaves unclear whether the explanation or wondering refers to the thing that happened to the patient (which may or may not be a behavioral event) or to the act that the (often unidentified) agent performed.

A passage is also uncodable when the context fails to clarify who the agent was or what exactly happened. For example, "By thinking of things you could understand them. But why in the square?' (Joyce, 1916/1993, p. 40).

\section{Dispositions}

Sometimes people ask questions such as "Why is he so shy?" This proposition is uncodable because it refers to a disposition rather than to a concrete behavioral event. By contrast, consider "Why is he so shy when the girls ask him out? Because he has never been on a date." This proposition is codable because it seems to refer to one or more instances of the agent's shy behavior. In short, dispositions are uncodable, whereas recurring behaviors are codable.

Statements that contain modal verbs such as "could" or "can" may be uncodable when they refer to a dispositional ability (or inability) to behave in a particular way. For example, in wondering " why I can't get rid of my accent," the speaker seems to refer to a disposition in himself rather than to a circumscribed psychological event. By contrast, in won- dering "why I could not fill out my tax forms correctly," the speaker refers to a behavioral event that actually took place.

\section{Claim Backings}

Sometimes the word because is used to give evidence for a claim being made rather than to explain a behavioral event. "It seemed like he was doing all right, 'cause the teacher didn't say anything to him." The clause beginning with " "cause"' backs up an inference ("It seemed . . .") through evidence; it does not explain why he was doing all right. Or consider "I think you are wrong here, because they have strict rules about this." The speaker is not explaining why a thought occurred in her, but she tries to provide evidence for her claim that the addressee is wrong. Evaluative statements typically are followed by a claim backing (e.g., "It's good that he left early because . . . ". "It does make life more interesting because. . .," and "It is essential to do that because ...").

A variation of claim backings are normative uses of "why" and "because." For example, the question "Why should I help him to relieve himself?" expresses a normative conflict rather than a need for an explanation. Similarly, "He should call her because she is waiting for him" does not explain a behavioral event but backs a normative proposition. Note, however, that the verb should can refer to actual events, especially in formal English: "He read one [novel] every six months, he said. And why should that make Charles Tansley angry?" This question expresses a true wondering about a psychological event, namely Tansley's getting angry.

Two other variations of claim backings are predictions and hypotheticals. Predictions do not explain behavioral events because no event has occurred yet, as in "He may never work again because he has schizophrenia." Hypotheticals, too, fail to refer to an actual behavioral event: "We would have to give them time to read before the exam because one hour is just too short."

Received November 30, 1995

Revision received June 13, 1996

Accepted June 28, 1996 